\title{
THEORETISCHE PHASEN DER (WEST)DEUTSCHEN GERMANISTIK IN DER NACHKRIEGSZEIT
}

\author{
JAN PAPIÓR
}

Der Einfluss der Nazizeit auf die „,deutsche Wissenschaft” - ,die aus dem mythisch-nationalen Geist der Romantik" " hervorging - war verheerend. Die Germanistik wurde schon im 19. Jahrhundert von vielen Wissenschaftlern (unter diesen befanden sich vor allem Germanisten) als ethnozentrische Disziplin verstanden, wahrscheinlich in der Folge einer Bestimmung des ersten deutschen Professors der Germanistik, Wilhelm Scherers, der die Aufgabe der Germanistik als „System nationaler Ethik" definierte und in diesem Sinne seine vielgelesenen Publikationen und Vorträge verfasste. Wilhelm Scherer stand unter dem Einfluss der Beratungen und Ergebnissen der Germanistentagung, die 1847 in Lübeck aus der Inspiration und unter der Leitung von Jacob Grimm und Teilnahme von 170 Wissenschaftlern durchgeführt wurde. Weil viele der Teilnehmer in die Frankfurter Nationalversammlung gewählt wurden, kann man die Behauptung wagen, dass schon in dieser Zeit von einer ,nationalen Gesinnung” ausgegangen werden kann.

Dies führte in der direkten Nachkriegszeit nach 1945 dazu, dass sich die führenden Germanisten im Westen des ehemaligen III. Reiches, die nicht selten auf eine latente, oder auch aktive Akzeptanz des Nazi-Regimes zurückblicken ${ }^{2}$ konnten und

\footnotetext{
${ }^{1}$ Ralf Schnell: Geschichte der deutschsprachigen Literatur seit 1945; Stuttgart 2005, S. 51.

${ }^{2}$ Viele führende Germanisten waren Mitglied der NSDAP, Sympathisanten der Nazi-Herrscher, oder sicherten ihre eigne Karriere auch als Mitläufer ab. Schon 1926 fordert der Leipziger Hochschulfunktionär und Germanist Walter Hofstaedter „«mit Entschiedenheit die Wissenschaft vom deutschen Wesen, die Deutschwissenschaft» als kennzeichnenden Oberbegriff für Germanistik” einzuführen. Von
} 
in der Konsequenz in einer theoretischen, aber doch ahistorischen Lehr- und Forschungspraxis verschanzten. Der Schweizer Germanist Walter Muschg (1898-1965) formuliert in seinem Buch Die Zerstörung der deutschen Literatur - Tragische Literaturgeschichte (1948) schon sehr früh eine scharfe Verurteilung der „,deutschen Wissenschaft” zur Nazi-Zeit: „Die Germanistik ist eine Komplizin des Verbrechens”.

Einer dieser namhaften Germanisten und Universitätsprofessoren war Wolfgang Kayser (1906-1960), der mit seinem programmatischen Handbuch - von den Studenten nach dem blauen Umschlag ironisch als „blaue Bibel” benannt - und zugleich seinem wichtigsten Werk Das sprachliche Kunstwerk (1947) die erste Phase der westdeutschen Germanistik begründetet und über knapp zwanzig Jahre beherrschte. Mit diesem ahistorischen Handbuch wird die «immanente Literaturwissenschaft» zum theoretischen Ausbildungskanon vieler westdeutscher Germanisten der frühen Nachkriegszeit. Unter diesen Voraussetzungen entwickelt sich auch die Literaturkritik in den Periodika.

Völlig anders entwickelte sich die theoretische Grundlage der Germanistik im östlichen Teil des ehemaligen III. Reiches. Schon durch die Rückkehr vieler Exilanten in den östlichen Teil Deutschlands, u. a. des Literaturwissenschaftlers Hans Mayer (1907-2001), sowie durch den Einfluss der sowjetischen Kultur- und Wissenschaftspraxis, war ein anderer Ausgangspunkt gegeben. Die Germanistik der DDR wird sich unter dem Einfluss des Marxismus und des «sozialistischen Realismus» entwickeln. Mit einem Begriff wäre die theoretische Position der ostdeutschen Germanistik $^{3}$ als «marxistische» oder auch «materialistische Sprach- und Literaturwissenschaft» zu umschreiben, über die später ausführlicher zu sprechen sein wird.

Die zweite Phase der westdeutschen Germanistik beginnt mit dem Germanistenkongress des Jahres 1966, als „eine Gruppe jüngerer Germanisten”, u. a. Karl Otto Conrady, Eberhard Lämmert, Walther Killy, Peter von Polenz (und weitere) die bisherige Forschungsgrundlage und praktizierten Studienpläne der etablierten Germanistik einer vernichtenden Kritik unterzogen. Infolge dieser Kritik, die ihre Ausformulierung in zwei wichtigen Buchveröffentlichungen ${ }^{4}$ findet, werden Forschung und Studium der westdeutschen Germanistik - wie dies Ralf Schnell formuliert „Anfang der siebziger Jahre einer Reihe gravierender und tiefgreifender Verände-

den Germanisten, die sich mit dem Naziregime in irgendeiner Weise liierten, wären - u. a. - zu nennen: Herbert Cysarz, Wolfgang Kayser, Fritz Martini, Josef Nadler, Julius Petersen, Hans Ernst Schneider, Erich Trunz, u. a. m. Über H.E. Schneider wurde auch eine satirisch-kritische Oper in Heidelberg inszeniert Der tausendjährige Posten oder der Germanist (2012), mit dem Libretto von Elfriede Jelinek.

${ }^{3}$ Es wäre an dieser Stelle nur auf die 13 Bände umfassende Geschichte der deutschen Literatur (die Reihe beginnt 1965 zu erscheinen) zu verweisen. Noch im Jahre 1989 (also kurz vor der sog. Wende) erscheint das Wörterbuch der Literaturwissenschaft, dessen Herausgeber - Klaus Träger - einer der profiliertesten Germanisten der ehemaligen DDR war. Auch in diesem Wörterbuch lässt sich die sozrealistische Grundlage (obwohl schon einigermaßen verwässert) problemlos feststellen.

${ }^{4}$ Ansichten einer künftigen Germanistik, hg. v. Jürgen Kolbe; Frankfurt/M. 1969. Neue Ansichten einer künftigen Germanistik, hg v. Jürgen Kolbe; München 1975. 
rungen des Faches" unterzogen, ,die sich aus unterschiedlichen, sogar widersprüchlichen (marxistischen, sozialkritischen, positivistischen, reformpädagogischen) Argumentationshorizonten speisten" . Diese neuen Argumentationshorizonte werden zu einer Inflation theoretischer Forschungs- und Studienansätze und damit zu einer Umorientierung der westdeutschen Germanistik führen. Diese lässt sich mit den folgenden Thesen umschreiben. Es kommt zu:

- „einer deutlich marxistisch begründeten sozialgeschichtlichen Orientierung;

- einer Zunahme des linguistisch-sprachwissenschaftlichen Anteils in Forschung und Lehre;

- einer - hiermit einhergehenden - Zurückdrängung der Mediävistik bzw. eine nachhaltige, sozialgeschichtlich orientierte Reform der Altgermanistik;

- einer Erweiterung des Literaturbegriffs zum Textbegriff, der auch Trivialwerke (Comics, Groschenhefte) und kommunikationsästhetische Aspekte von Literatur einbezog;

- einer verstärkten Hinwendung zu Fragestellungen relevanter Praxisfelder der Germanistik (Schule); [sowie]

- einer Öffnung der Literaturwissenschaft auch für Aspekte der audiovisuellen Medien"6.

Mit dieser Neuorientierung werden Problematisierungen und Fragenkomplexe geöffnet, die in den früheren germanistischen Forschungen und Studienplänen nicht denkbar waren und aus diesem Grunde nicht berücksichtigt wurden und auch nicht berücksichtigt werden konnten. Ein weiterer Aspekt, der von R. Schnell nicht besonders hervorgehoben wurde, ist die theoretische Forschungsgrundlage, die sich seit den späten sechziger Jahren grundsätzlich ändert.

Schon in den sechziger Jahren tritt Hans Norbert Fügen mit zwei wichtigen Buchveröffentlichungen zur Literatursoziologie ${ }^{7}$ an die wissenschaftliche Öffentlichkeit. Immerhin war diese Richtung schon seit Jahrzehnten in der deutschen wissenschaftlichen Forschung ${ }^{8}$ anwesend, aber dieser Paradigmenwechsel macht im kulturpolitischen Kontext auf andere Forschungsgrundlagen vehement aufmerksam. Das Ergebnis dieses Neuansatzes, der zugleich eine Absage an die Konzeption W. Kaysers bedeutete, bringt eine Inflation der theoretischen Grundlagen und Modelle, wie Literatur und Text wissenschaftlich untersucht werden können. Den Paradigmenwechsel verzeichnet auch die Folge der zwei größten Reallexiken zur deutschen Literaturgeschichte und Literaturwissenschaft. Wenn man sich noch in den

\footnotetext{
${ }^{5}$ R. Schnell, S. 52.

${ }^{6}$ R. Schnell, S. 52.

${ }^{7}$ H. N. Fügen: Die Hauptrichtungen der Literatursoziologie und ihre Methoden; Bonn 1964. Wege der Literatursoziologie, hg. von H. N. Fügen; Berlin 1968

${ }^{8}$ Es wäre auf die Publikation Samuel Lublinskis (Litteratur und Gesellschaft im 19. Jahrhundert, 1899-1900), Lewin L. Schückings (Die Soziologie der literarischen Geschmacksbildung, 1923), und Arnold Hausers (Sozialgeschichte der Kunst und Literatur, 1951) hinzuweisen.
} 
Jahren 1958-1988 für eine Herausgabe unter geringfügiger Neubearbeitung des seit 1925 erscheinenden Reallexikons der deutschen Literaturgeschichte [RLG] entschloss, erscheint erst - als späte Konsequenz dieses Paradigmenwechsels - seit 1997 das Reallexikon der deutschen Literaturwissenschaft [RLW], in dem der neuste Stand der theoretischen und terminologischen Grunddefinitionen der germanistischen Literaturwissenschaft erfasst wird. Es ist markant, dass man sich erst ganz zum Ende des 20. Jahrhunderts für eine terminologische Modifizierung entschließen konnte und anstatt «Literaturgeschichte» (1925ff., 1958ff.) in der neuen Bearbeitung «Literaturwissenschaft» (1997) setzt, obwohl diese doch schon seit langem fällig war - etwa seit W. Scherers Poetik (posthum 1888; erneut 1977).

Es wird nun nach diesem RLW die Folge der wichtigsten neuen Forschungskonzeptionen der deutschen Literaturwissenschaft angeführt. Allen definierenden Beiträgen voran sei erwähnt die

> „Literaturwissenschaft” [II: 485-489] (es wird im RLW nur angeführt, das diese eine „Wissenschaft [ist], deren Gegenstand die Literatur ist”, es fehlt jedoch eine Anführung der Teilbereiche, die diese Lw wissenschaftswissenschaftlich konstituieren, wie: diachronisch geprägte Überlegungen zum Gegenstand, Literaturgeschichte, Theorie der Literatur, Methodologie und Bibliographie; in diesem Zusammenhang wäre von einer dekonstruktivistischen Modellierung des RLW zu sprechen);

$>$,marxistische [materialistische] Literaturwissenschaft” [II: 541-546] (die Anfänge gehen bis auf K. Marx und F. Engels seit 1848 zurück, aber eine konstruktive und prospektive Entwicklung wird im deutschen Kultur- und Sprachraum erst von der DDR-Germanistik ${ }^{10}$ erreicht und steht unter dem Einfluss von F. Mehring, G. Lukács und der SED-Ideologie, die sowjetischer Prägung ist);

$>$ „Phänomenologische Literaturwissenschaft” [III: 62-64] (durch E. Husserl werden Untersuchungen angeregt, die - insbesondere in den Arbeiten von R. Ingarden ${ }^{11}$ seit 1931 - ästhetische Probleme in den Vordergrund der Untersuchung stellen und das Kunstwert als ,intentionales” Objekt verstehen, es wird insbesondere die Seinsweise des Kunstwerkes untersucht, in dem Unbestimmtheitsstellen entdeckt werden und das Verhältnis zur Realität in schematisierten Ansichten zusammenfasst);

\footnotetext{
${ }^{9}$ Hiermit wird der Beleg im Reallexikon der deutschen Literaturwissenschaft [RLW] abgesichert.

${ }^{10}$ Siehe hierzu die 3. Fußnote.

${ }^{11}$ R. Ingarden: Das literarische Kunstwerk. Eine Untersuchung aus dem Grenzgebiet der Ontologie, Logik und Literaturwissenschaft 1931.

In diesem Zusammenhang wäre darauf hinzuweisen, dass sowohl E. Staiger, als auch W. Kayser mit keinem Wort auf R. Ingarden verweisen, dessen theoretische Konzeption doch die - seinerzeit moderne -Position des literarischen Textes - im ontologischen Sinne - als Kunstwerk begründet. Auch der Hinweis darauf, dass R. Ingarden seine fundamentale Untersuchung im deutschen Göttingen un als Schüler von E. Husserl verfasste.
} 
„Werkimmanente Interpretation” [III: 834-837] (diese Richtung ist als Reaktion gegen die Totalisierung sozialer Strukturen in den 20. und 30. Jahren des 20. Jahrhunderts, und den Einbezug äußerer Implikationen in die Betrachtungsgrundlagen der Literatur zu verstehen: diese ist im deutschen Sprachraum mit den Konzeptionen des Schweizer Germanisten Emil Staiger ${ }^{12}$ und des deutschen Germanisten Wolfgang Kayser ${ }^{13}$ verbunden, alles was für die wissenschaftliche Betrachtung der Dichtung von Bedeutung wäre, soll nur aus dem ,sprachlichen Kunstwerk” - wie W. Kayser es formuliert - erkannt werden);

„Literatursoziologie” [II: 473-477] (die Ansätze dieser Betrachtungsform der Literatur lassen sich schon zum Anfang des 20. Jahrhunderts ${ }^{14}$ feststellen und stehen im Zusammenhang mit der Entwicklung und Autonomisierung der Soziologie als Forschungsrichtung; man richtet sich - bedeutend seit 1950 gegen ,irrationalistische” Tendenzen der deutschen Lw, unternimmt Versuche die Wertsphäre aus der Forschung auszugrenzen und mit empirischen Beweismethoden zu arbeiten, in der neueren Forschung wird die Literatur als Teilsystem der Soziologie gesehen);

$>$,Sozialgeschichte" [III: 454-458] (diese wird als „gesellschaftsbezogene Perspektive in der historiographischen Darstellung von Literatur" beschrieben und steht ebenfalls im Zusammenhang mit der Entwicklung der Soziologie als Forschungsdisziplin; seit 1951 werden bedeutender literarische und gesellschaftliche Verhältnisse, Beziehungen und Einflusspären erkundet um literaturgeschichtliche Entwicklungen zu beschreiben, besonders bedeutsam ist diese Forschungsrichtung für Institutionen des literarischen Lebens und hat eine Tradition, die bis ins 19. Jahrhundert zurückreicht; der Neuansatz dieser Richtung ist u. a. von A. Hausers Monographie ${ }^{15}$ mitbestimmt worden);

$>$ „Hermeneutik” [II: 25-33] („Theorie des Lesens, Verstehens und Interpretierens von Texten", die insbesondere durch H.-G. Gadamers ${ }^{16}$ Forschungen seit 1960 erneut aufgenommen wurden; die stehen unter dem Rückgriff auf W. Diltheys und F. Schleiermachers Forschungen und Bücher);

$>$ „Dekonstruktion” [I: 324-337] (seit den sechziger Jahren des 20. Jahrhunderts in der Konsequenz von Jacques Derridas Dekonstruktionstheorie ${ }^{17}$, die

${ }^{12}$ E. Staiger: Die Zeit als Einbildungskraft des Dichters. Untersuchungen zu Gedichten von Brentano, Goethe und Keller; 1939.

${ }^{13}$ W. Kayser: Das sprachliche Kunstwerk; 1948.

${ }^{14}$ Siehe die 8. Fußnote.

${ }^{15}$ Siehe die 8. Fußnote.

${ }^{16}$ Besonders Einflussreich war H.-G. Gadamers Buch Wahrheit und Methode. Grundzüge einer philosophischen Hermeneutik, 1960.

${ }^{17}$ J. Derridas Werke erscheinen seit den sechziger Jahren des 20. Jahrhunderts. Zu seinen wichtigsten und bekanntesten Werken gehören Grammatologie (1967, deutsch 1974) und Die Schrift und die 
auf M. Heideggers Philosophie zurückgreift, versteht sich als eine lw Dekonstruktion, also als eine Richtung, die gegen die Ermittlung von sinnzentrierten und traditionellen Interpretationen einwirken will; neben der französischen gibt es auch eine amerikanische Richtung, die auf Paul de Man's ${ }^{18}$ Theoriekonzeptionen zurückgeht);

$>$,Analytische Literaturwissenschaft” [I: 79-81] (wurde in den späten 60., 70. und 80. Jahren des 20. Jahrhunderts entwickelt; diese auf den theoretischen Konzeptionen der analytischen Philosophie aufbauende Konstruktion ${ }^{19}$ ist gegen die geisteswissenschaftliche und hermeneutische Tradition gerichtet und will sich gegen diese abgrenzen; es wird ,eine intersubjektiv nachprüfbare Basis" der Lw gefordert, ihre Vertreter verstehen sich als Wissenschaftler, die die Disziplin verwissenschaftlichen wollen);

$>$,Strukturalismus” [III: 535-539] (vorausgesetzt werden Semiotik und Textlinguistik; hiermit wird grundsätzlich jedes Kunstwerk, so auch Literatur als Zeichenstruktur interpretiert, setzt keine Theorien als wahr voraus, definiert alle konstruierten Untersuchungsobjekte als Strukturen und/oder Systeme und als mögliche Varianten oder Alternativen; die Geschichte der Richtung beginnt mit Ferdinand de Saussures linguistischer Theorie ${ }^{20}$, wird aber im deutschen Sprachraum erst seit den 60 Jahren des 20. Jahrhunderts rezipiert);

$>$,Literaturpsychologie” [II: 469-473] (eine Richtung, die Fragen an die Literatur aus einem psychologischen Kontetxt richtet, frühere Anfänge noch bei W. Dilhey (Das Erlebnis und die Dichtung, 1905), die Neuanfänge seit den frühen 70. Jahren, einerseits kann von einer psychoanalytischen Lw gesprochen werden, die auf S. Freuds und Jacques Lacans Theorien zurückgreifen, und andererseits von einer empirischen Literaturpsychologie gesprochen werden);

$>$ „Poststrukturalismus” [III: 140-144] (Richtung in der Philosophie und Lw, die sich seit den 1970-Jahren entwickelt, die als Kritik am Strukturalismus verstanden werden muss und eine Zerstreuung von Sinn als Ausgangspunkt der Überlegungen macht; verbunden hiermit ist eine Ablehnung der Grundthese, dass ein jeder Text einen Sinn vermittelt, insgesamt ist die Richtung transdisziplinär, obwohl Beiträge zu Poststrukturalismus jedoch schon seit

Differenz (1967). Eine Zusammenfassung seiner Gedanken bringt P. Engelmann in der Anthologie Jacques Derrida: Die différance - Ausgewählte Texte (2004).

${ }^{18}$ Seine erste Untersuchung, die diesen Problemen nachgeht ist Blindness and Insight, 1971. Sein nächste bedeutende Untersuchung erscheint 1979: Allegories of Reading (deutsch Allegorien des Lesens, 1989).

${ }^{19}$ Einen zusammenfassenden Überblick bietet die Publikation Analytische Literaturwissenshaft, hg. v. Peter Finke und Siegfried J. Schmidt (1984).

${ }^{20}$ Cours de linguistique générale (1916), deutsch Grundfragen der allgemeinen Sprachwissenschaft (1967). 
den 60 Jahren vorliegen; die Richtung ist mit J. Derridas Text ${ }^{21}$ aus dem Jahre 1967 verbunden; in der Lw wird der Poststrukturalismus oft auch als Dekonstruktion bezeichnet und bewirkt eine Neubesinnung auf die rhetorischen Grundsätze eines Textes);

$>$,Rezeptionsästhetik” [III: 285-288] (eine sich seit den frühen siebziger Jahren entwickelnde Betrachtungsweise der Literatur, die „Theorie, Analyse und Geschichte der Beziehung zwischen Text und Leser" zu erfassen versucht, wobei ein Interaktionsverhältnis postuliert wird; diese steht in einem Zusammenhang mit Rezeptionsforschung und Literatursoziologie und wird im deutschen Sprachraum intensiviert durch die Untersuchungen Wolfgang Isers ${ }^{22}$ );

$>$ „Diskurstheorie” [I: 372-374] (Forschungszugänge, die sich der Untersuchung von Äußerungszusammenhängen widmen und wie Äußerungen konstruiert werden, es gibt philosophische, linguistische, wie auch lw Diskursanalysen; diese gehen auf M. Foucaults, A. J. Greimas, J.-F. Lyotard und J. Habermas Theorien zurück);

$>$ „Feministische Literaturwissenschaft" [I: 575-577] (nachdem sich das Adjektiv „feministisch” seit den 1970-ger Jahren durchsetzte, konnte sich auch eine f. Lw entwickeln, die von Frauenbewegungen in den USA und Frankreich in der BRD angeregt wurde, vorausgehend war die Frauenliteratur, feminine Essayistik, die zu theoretischen Überlegungen führten, insbesondere Wertungskriterien, einen feministischen Literaturkanon und führt zu einer Berücksichtigung weitgehend vernachlässigter Formen, Brief, Tagebuch autobiographische Erzählung, in denen sich Autorinnen bevorzugt ausdrückten);

> „Psychoanalytische Literaturwissenschaft” [III: 191-194] (Texte, Autoren und Leser werden mit tiefenpsychologischen Kategorien untersucht und konzentriert sich auf psychische Phänomene konkreter Texte, die Richtung geht auf S. Freud zurück, der selbst Analysen literarischer Texten vorgelegt hat);

$>$ „Empirische Literaturwissenschaft” [I: 443-446] (es werden erfahrungswissenschaftliche Methoden angewendet um „Bedingungen und Prozesse literarischen Handelns" zu erforschen und um sich durch diesen Zugang von hermeneutischen Forschungspraktiken abzugrenzen, ein Buch unter dem Titel Grundriss der empirischen Literaturwissenschaft wurde 1980 von S.J. Schmidt vorgelegt).

Der Einblick in die Vielfalt der theoretischen Konzepte literaturwissenschaftlicher Erkenntnis, die sich seit den 60-ger Jahren des 20. Jahrhunderts entwickelt haben ist bedrückend und wirft die Frage nach der wissenschaftstheoretischen wie auch kulturhistorischen Relevanz der einzelnen Modelle auf. Es sei auf das Grund-

\footnotetext{
${ }^{21}$ Siehe die 17. Fußnote.

${ }^{22}$ Insbesondere wäre auf die Untersuchungen Der implizite Leser [...], 1972 und Der Akt des Lesens [...], 1976 zu verweisen.
} 
anliege dieser Entwicklungen hinzuweisen, die - im Grunde - durch Bemühungen um eine Verwissenschaftlichung der Literaturwissenschaft angeregt wurden.

Man konnte und wollte (1.) sich nicht mit den philologischen, hermeneutischen, geistesgeschichtlichen, phänomenologischen, werkimmanenten Grundlagen der kultur- und literaturwissenschaftlichen Explanation zufriedenstellen, weil die Art der Zugangsweise sich zu sehr von den naturwissenschaftlichen Disziplinen unterscheidet. Dies könnte ein Hinweis darauf sein, dass man unberechtigt den Gegenstand der Forschungen in beiden Bereichen (Natur-Kultur) zu identifizieren versuchte, der seinen Ausdruck in der Nomothetisierung ${ }^{23}$ und Idiographisierung ${ }^{24}$ der Wissenschaftsbereiche fand. Es ist relevant, darauf hinzuweisen, dass der Mensch als Individuum vor allem durch seine ontologischen und epistemologischen Fähigkeiten zu seiner kulturhistorisch determinierten Wesenseigenart vorstoßen kann. Was für Werkzeuge er sich dazu herstellt ist von nur marginaler Bedeutung.

Es ist (2.) nicht zu übersehen, dass in den meisten Fällen die Erweiterung oder Neubenennung der theoretischen Vorschläge auf adjektivischen Formulierungen oder substantivierten Adjektivformulierungen aufbaut. Hiermit wird also die wissenschaftswissenschaftliche Definition der Literaturwissenschaft nicht problematisiert oder negiert, sondern nur durch eine neue Zugangsweise modifiziert. Viele dieser Vorschläge wären im Grunde nur als modifizierte literaturhistorische und/oder literaturmethodologische, ev. literaturtheoretische Betrachtungsweisen literarischer Texte zu werten, die immerhin einen relevanten Einfluss auf die literaturkritische und literaturwissenschaftliche Praxis hatten oder immer noch haben.

In einigen Fällen bezieht sich die Modifizierung (3.) in der Art und Weise von Rückgriffen auf Modelle und Zugangsweisen anderer Wissenschaftsbereiche, wie z. B. der Anthropologie, Ästhetik, Kulturwissenschaft, Philosophie, Psychologie, und Soziologie. Der Gegenstand und die Zugangsweise zum Gegenstand (L), wie auch seine Konzeptualisierung ist in diesen Forschungsbereichen jeweils ein anderer, als in der traditionell ${ }^{25}$ verstandenen und realisierten Literaturwissenschaft. In wieweit solche Übernahmen produktiv sein können und den Gegenstand der literaturwissenschaftlichen Forschung nicht beeinträchtigen, sollte entschiedener überprüft werden. Analog zur Untersuchung des Wassers in der Physik und Chemie, die eine andere Konzeptualisierung der Erforschung desselben Gegenstandes realisieren, kann auch der literarische Text in anderen Forschungsbereichen, mit jeweils anderen Werkzeugen befragt werden.

\footnotetext{
${ }^{23}$ Die Forschung wird durch Regeln geleitet sowie soll Regeln erkennen. Dieser Grundsatz bezieht sich nur auf die Naturwissenschaften.

${ }^{24}$ Die Forschung wird in mehr oder weniger exakten Beschreibungen und Nachempfindungen realisiert. Dieser Grundsatz bezieht sich nur auf die Kulturwissenschaften.

${ }^{25}$ Diese adjektivische Klassifizierung soll als wissenschaftswissenschaftliche Grundlage verstanden werden.
} 
Es ist (4.) nicht zu übersehen, dass in den soeben erwähnten Modellen das ,literarische Kunstwerk” (R. Ingarden), oder das „,sprachliche Kunstwerk”26 (W. Kayser) nicht immer als «Kunstwerk» eingesetzt, sondern als anthropologische, ästhetische, kulturelle, philosophische, psychologische, und soziologische Realität betrachtet wird und aus dieser Perspektive untersucht wird. Wobei unberücksichtigt bleibt, oder zu mindestens marginalisiert wird, dass dieses Kunstwerk eine Konstruktion einer konkreten Person ist, die mit einem Kulturbewusstsein verbunden ist und eine eigenartige Auffassung darstellt. Dass das literarische oder sprachliche Kunstwerk auch diese Eigenschaften aufweisen kann, ist nicht zu bezweifeln. Aber der Schriftsteller schreibt sein Werk (ob bewusst oder unbewusst sei dahingestellt) nicht als anthropologisches, ästhetisches, kulturelles, philosophisches, psychologisches, und soziologisches Werk, sondern nur als «literarisches» Kunstwerk, nicht einmal als rein sprachliches Kunstwerk.

Die Problematik des Kunstwerkes liegt (5.) in seiner Seinsweise: ob diese Konstruktion einen realen Bezug aufweist und hiermit einen tatsächlich-realen Zustand dokumentiert, oder ob diese Konstruktion in bewusster Weise sich von diesem Bezug distanziert und eine fiktionale Wirklichkeit prägt, die zwar in Einzelheiten reale Bezüge aufweisen kann, aber als ganzheitliche und vom Schriftsteller als abgeschlossene Konstruktion projektiert (auch wenn diese im dekonstruktiven Sinne eine offene Struktur aufweist), ist doch nur eine fiktionale, also in Wirklichkeit nichts Reales dokumentierende Darstellung ${ }^{27}$. Diese Problematik liegt halt auf einer anderen und zugleich tieferen Ebene.

Es wurde in den letzten Jahrzehnten im Zuge dieser Vielfalt - angeregt durch diese Modelle - die fiktionale Seinsweise der literarischen Kunstwerke erneut befragt. Als Textprodukt ist seine Seinsweise zwar eine Realität, die man als Buch oder Textproduktion auch auf der Bildschirmseite erwerben kann, aber als intellektuelles oder literarisch-künstlerisches Produkt und Aufzeigung eines kulturphilosophischen Sinnes hat das literarische/sprachliche Kunstwerk keine Entsprechung in real-wirklichen Konstellationen, es ist also weiterhin eine fiktionale Projektion oder Konstruktion und Vision des Verfassers. Hiermit wäre dann darauf zurückzukommen, das eines der Grundphänomene dieser Form der Kunstwerke ihr fiktionaler Charakter ist, der jedoch nicht problemlos auf nur anthropologische, ästhetische, kulturelle, philosophische, diskursive, psychologische, und soziologische Phänomene reduziert werden kann.

Es wäre folglich (6.) zu fragen, was für einen Bezug das literarische/sprachliche Kunstwerk dokumentiert? Es ist einerseits der Bezug, genauer die Sprache, in der es

\footnotetext{
${ }^{26}$ Es soll auf die verblüffende Ähnlichkeit der Begriffe hingewiesen werden. Dass die Bedeutung des „Literarischen” für den Gegenstand der Lw gewichtiger zu sein scheint, wurde bis heute nicht entsprechend gewertet. Das „Sprachliche” spielt im Leben des Menschen und allen Bereichen der Kulturwissenschaften eine bedeutende Rolle, bringt aber das „Literarische” alleine noch nicht zur Geltung.

${ }^{27}$ Wenn auch der Text als materielles Objekt real funktioniert.
} 
realisiert wird. Andererseits ist es der Bezug zum Kulturgedächtnis, sowie zur Kultur und Kulturgeschichte einer sozialen Gruppe (Nation), und nicht zuletzt der werkliterarische Bezug, der mit fiktionalen Modellen und Systemen realisiert wird.

Eines der Grundprobleme dieser Kunstwerke wäre (7.) die Sprache, in welcher der Schriftsteller sein Werk realisiert. Die Problematik ist deswegen so virulent, weil wir den Unterschied zur Sprache des Alltags, der Umgangssprache (insbesondere in modernen zeitgenössischen Werken) nur indirekt und mit Schwierigkeit erkennen können. Noch im Mittelalter war die Alphabetisierung, das Lesen und Schreiben eine Kunst. Seit etwa hundert Jahren ist dies (zwar mit geringfügigen Ausnahmen) kein Problem mehr. Aber deswegen wird die Erkenntnis und Betrachtung des Kunstwerkes immer problematischer und ihr ästhetischer Wert immer demokratischer. Zudem wäre die Umstellung von drucktechnischen Formen (Bücher, allgemeiner Texte) auf elektronische Formen (Internet, Digitalisierung) zwar ein demokratisierendes Signum unserer Zeit, aber dennoch ein beschränktes, weil auch in den elektronischen Formen die tradierten Sprachen der Grundstock der Kommunikation sind. 